\title{
Vortices near barriers with multiple gaps
}

\author{
By E. R. JOHNSON AND N. ROBB McDONALD \\ Department of Mathematics, University College London, Gower Street, \\ London WC1E 6BT, UK
}

(Received 26 August 2004 and in revised form 3 December 2004)

Two models are presented for the motion of vortices near gaps in infinitely long barriers. The first model considers a line vortex for which the exact nonlinear trajectories satisfying the governing two-dimensional Euler equations are obtained analytically. The second model considers a finite-area patch of constant vorticity and is based on conformal mapping and the numerical method of contour surgery. The two models enable a comparison of the trajectories of line vortices and vortex patches. The case of a double gap formed by an island lying between two headlands is considered in detail. It is noted that Kelvin's theorem constrains the circulation around the island to be a constant and thus forces a time-dependent volume flux between the islands and the headlands. When the gap between the island and a headland is small this flux requires arbitrarily large flow speeds through the gap. In most examples the centroid of the patch is constrained to follow closely the trajectory of a line vortex of the same circulation. Exceptions occur when the through-gap flow forces the vortex patch close to an edge of the island where it splits into two with only part of the vortex passing through the gap. In general the part squeezing through a narrow gap returns to near-circular to have a diameter significantly larger than the gap width.

\section{Introduction}

The abyssal ocean consists of disjoint basins separated by mid-ocean ridges, many of which are not perfect barriers but are punctuated by gaps. Recognizing the important role that gaps in mid-ocean barriers may play in ocean circulation, several authors have sought to model the effects of gaps on various ocean processes. These studies include, for example, the flow of stratified abyssal ocean in the presence of a partial meridional (north-south) barrier (Pedlosky 1994), the passage of a western boundary current across a gap (Sheremet 2001) and the rotating exchange flow through a narrow gap between large-scale ocean basins (Nof 1995). Motivated by the topographic complexity of features like the Mid-Atlantic Ridge and the Indonesian Archipelago, Pratt \& Spall (2003) discuss linear barotropic wind-driven flow through a porous ridge, their idea being that a porous ridge is able to impede, but not totally block, incoming currents. By considering the flow about each island comprising the ridge, Pratt \& Spall (2003) were able to determine the magnitude and structure of the zonal flow through the ridge. Since ocean vortices can propagate large distances it is likely that abyssal eddies, which have been observed to exist at a depth of $4000 \mathrm{~m}$ (McWilliams 1985), will frequently encounter mid-ocean ridges occurring at similar depths (e.g. the Walvis Ridge in the South Atlantic) and as vortices also play a significant role in the transport of mass, momentum, heat and salt in the global ocean circulation, an understanding of the circumstances under which they are able to penetrate gaps in ridges is important. Richardson \& Tychensky (1998) presented observations of the 
mid-depth ocean showing how the presence of seamounts can severely disrupt the passage of Meddies. In some cases, Meddies were observed to bifurcate upon collision with a seamount. Dewar (2002) modelled this process as a 'cloud' of baroclinic point vortices interacting with a circular cylinder extending throughout the fluid column, the redistribution of the vortices within the 'cloud' upon collision with the seamount being representative of the deformation of a continuous eddy. Dewar (2002) also described an iterative numerical method enabling the collision of the vortex cloud with multiple seamounts to be computed. Wang \& Dewar (2003) subsequently used this method to study the interaction of a cloud of vortices with two seamounts, with the aim to assess the role played by Meddies in maintaining the Mediterranean Salt Tongue. In addition to abyssal vortices, surface-trapped vortices may also encounter partial (or porous) barriers. This occurs, for example, in the collision of North Brazil Current rings with the Lesser Antilles (Fratantoni, Johns \& Townsend 1995). Simmons \& Nof (2002) have studied encounters of vortices with barriers having either a single or many gaps. In particular they used both analytical methods based on integral constraints, and the numerical solution of the primitive equations to study a lens propagating under the $\beta$-effect or large-scale advection toward a gap (or gaps). They found that, depending on its strength, a vortex could squeeze though a gap unchanged, or be partially drained by wall currents until of size comparable to the gap and then pass through.

This work investigates the behaviour of vortices near gaps in an infinitely long wall in the context of the two-dimensional Euler equations (valid at arbitrary, but constant, rotation rates for a constant-depth barotropic ocean), obtaining exact analytical expressions for the fully nonlinear trajectories of line vortices and computing accurately the motion of finite-area vortex patches. In the absence of background flows (and other effects such as the $\beta$-effect), a vortex far from any gaps (compared to its distance from the wall) propagates parallel to wall as though driven by an image in the wall. If the vortex then encounters a gap its behaviour is determined by the ratio of the gap width to the distance of the vortex from the wall. Vortices jump narrow gaps and pass through wide gaps. Johnson \& McDonald (2004a) use Routh's rule (Routh 1881; Saffman 1992) for the transformation of the line vortex Hamiltonian under conformal mapping to obtain the exact trajectory of a point vortex moving along a straight wall in which there is a single gap, showing that the vortex jumps the gap if the gap width is at least twice the distance of the vortex from the wall far upstream and passes through narrower gaps. This result is compared to the trajectory of the centroid of a vortex patch of uniform vorticity computed numerically using a combination of conformal mapping and contour dynamics (Dritschel 1988; Zabusky, Hughes \& Roberts 1979). Provided the vortex patch remains close to circular its trajectory is well-modelled by that of an equivalent line vortex. For many cases, especially those in which the vortex moves due to the interaction with the boundary alone (i.e. it is not advected by an ambient flow) the patch stays close to circular and is, consequently, very well-modelled by a line vortex.

Johnson \& McDonald $(2004 b, 2005)$ describe the interaction of vortices with two circular islands and with a group of point islands. The flow domain is no longer simply connected and thus the circulation about the islands must be specified at each instant. Discussing the flow past an offshore island, Johnson (1989) points out that, since the island boundary is a material surface, Kelvin's theorem requires the circulation about an island to be constant for all time. For flow to be at rest near the islands when vortices are far away the circulation about the islands is initially zero and so it remains zero. As noted in Johnson (1989) and Johnson \& McDonald 
(2004b) this means that there is a time-dependent round-island volume flux given by a time-dependent but spatially constant streamfunction value on each island determined by the constraint that the circulation around the island vanishes. Many remarkable consequences of this result for ocean basin-scale flows have subsequently been noted (Pedlosky et al. 1997; Pedlosky \& Spall 1999; Helfrich, Pedlosky \& Carter 1999; Pedlosky 2000, 2001a, $b$; Wells \& Helfrich 2001). As it is the circulation constraint that determines the more extreme motions below, it is perhaps of interest to note a description in physical terms. Since, unlike other material circuits in the flow, the material band formed by the fluid elements against an island cannot deform (but can be broken if the flow separates as noted in $\S 5$ ), the angular momentum of the band about any fixed point is well-defined. In inviscid flow fluid elements exert no shear stress on each other and so the angular momentum of the band is conserved, and is zero for flow forced by vortices approaching from large distances. As a vortex approaches an island the pressure field of the vortex causes elements in the band and nearest to the vortex to begin moving in the direction of the circulation round the vortex as shown below in figure 6 . To conserve the angular momentum of the band, those elements against the island but further away from the vortex begin to move in the opposite direction. When this opposing motion is forced through a narrow gap large velocities are generated.

Section 2 derives the conditions that govern the streamfunction for the flow in a general domain consisting of two subdomains sharing a common boundary consisting of an island chain between two headlands and the particular form for collinear gaps. Vortex patch computations are reduced through symmetry and consideration of unbounded flows to finding at each instant an irrotational flow with vortex sheets of known strength across the gaps. A rapid and accurate method for finding this flow for two collinear gaps is obtained by conformal mapping. Section 3 describes line vortex motion by mapping the flow to a doubly periodic rectangle and thus is closely related to classical problems of the motion of a lattice of vortices (Tkachenko 1966; Benzi \& Legras 1987; Campbell, Doria \& Kadtke 1989; O’Neil 1989; Weiss \& McWilliams 1991; Stremler \& Aref 1999). The circulation constraint however introduces significant differences and difficulties which can be circumvented by a particular choice of cuts associated with the complex potential of the vortices. The remarkable flow fields associated with vortices near narrow gaps are described and line vortex trajectories given. Section 4 presents numerical integrations for finite-area vortex patches, showing that provided patches do not approach the central island too closely they follow closely the paths of equivalent line vortices. Patches that strike the island can either return to symmetry and again follow line vortex trajectories or be torn apart with the strong through-gap flow forcing significant portions of large vortices through small gaps. The results are discussed briefly in $\S 5$ where the immediate extensions to asymmetrically located islands, finite-width islands and islands in channels are noted. An appendix extends the result of Johnson \& McDonald (2004a) on the relationship between patch centroids and line vortices to show that, even in the presence of arbitrarily strong boundary effects, patch centroids travel with the same velocity as the equivalent line vortices.

\section{General considerations}

Consider the motion of an incompressible inviscid fluid in a two-dimensional domain $\mathscr{D}$ that can be decomposed into two subdomains $\mathscr{D}_{1,2}$ as in figure 1 . The subdomains $\mathscr{D}_{1}$ and $\mathscr{D}_{2}$ are separated by a gap between two headlands $\mathscr{H}_{1}$ and $\mathscr{H}_{2}$ 


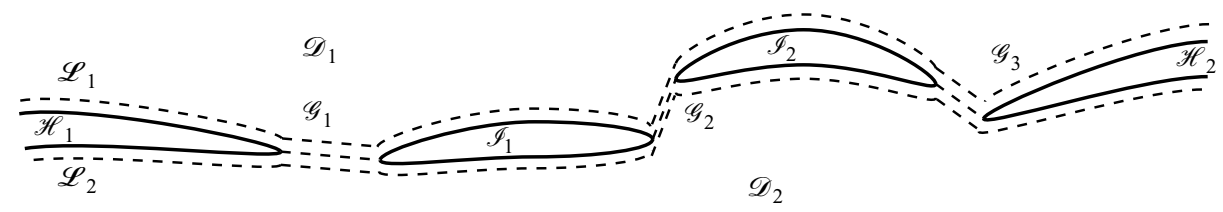

FIGURE 1. The flow region with domains $\mathscr{D}_{1,2}$ separated by a gap between headlands $\mathscr{H}_{1,2}$ with the opening further interrupted by islands $\mathscr{I}_{1}, \mathscr{I}_{2}, \ldots, \mathscr{I}_{M-1}$, breaking the region between the headlands into smaller gaps $\mathscr{G}_{1}, \mathscr{G}_{2}, \ldots, \mathscr{G}_{M}$. Here $M=3$. The line $\mathscr{L}_{1}$ passes along the $\mathscr{D}_{1}$ sides of the islands and headlands and $\mathscr{L}_{2}$ along the $\mathscr{D}_{2}$ side. The lines $\mathscr{L}_{1,2}$ coincide across the gaps.

and the gap is further interrupted by islands $\mathscr{I}_{1}, \mathscr{I}_{2}, \ldots, \mathscr{I}_{M-1}$, breaking the region between the headlands into smaller gaps $\mathscr{G}_{1}, \mathscr{G}_{2}, \ldots, \mathscr{G}_{M}$. The case of no islands $(M=1)$ is described in Johnson \& McDonald (2004a). If one of the headlands is absent, the geometry becomes that of a string of offshore islands with the case of a single offshore island described in Johnson \& McDonald (2004b) and multiple islands in Johnson \& McDonald (2005). Suppose that finite-area vortices lie in both $\mathscr{D}_{1}$ and $\mathscr{D}_{2}$. Following Johnson $\&$ McDonald (2004a), let the streamfunction induced by the vortices in the region $\mathscr{D}_{1}$ and satisfying the no-normal-flow boundary condition along the line $\mathscr{L}_{1}$ (composed of the gaps joined along the $\mathscr{D}_{1}$ sides of the islands and headlands), and specified conditions at infinity, be $\psi_{1}$, so

$$
\psi_{1}=0 \quad \text { on } \mathscr{L}_{1} \text {. }
$$

Similarly let the streamfunction induced by the vortices in the region $\mathscr{D}_{2}$ satisfying the no-normal-flow boundary condition on $\mathscr{L}_{2}$ and given far-field conditions be $\psi_{2}$, so

$$
\psi_{2}=0 \quad \text { on } \quad \mathscr{L}_{2}
$$

Denote the total streamfunction induced by the all vortices in the region $\mathscr{D}=\mathscr{D}_{1} \cup \mathscr{D}_{2}$, and satisfying the no-normal-flow boundary condition on $\partial \mathscr{D}$ (or with specified irrotational flow at infinity if either $\mathscr{D}_{1}$ or $\mathscr{D}_{2}$ extends there), by $\Psi$. Since the islands and headlands are impermeable, $\Psi$ on each island or headland boundary is independent of position and simply a function of time. The difference between these values gives the time-varying volume flux through the gaps. In particular the difference between the values of $\Psi$ on the two headlands gives the net volume flux between the domains $\mathscr{D}_{1}$ and $\mathscr{D}_{2}$. If either $\mathscr{D}_{1}$ or $\mathscr{D}_{2}$ is a closed basin then this flux vanishes. This will be taken to be the case here so $\Psi$ is the same on each headland and, without loss of generality, can be taken as zero there. The inclusion of a net volume flux between the domains is straightforward and the interesting flow patterns that arise are discussed in Johnson \& McDonald (2004a) for the case of an uninterrupted gap. By its impermeability, the boundary of each island is a material surface throughout the motion and so Kelvin's theorem requires the circulation around each boundary to be a constant of the motion. This constant is determined here by supposing that all vortex patches originated far from the origin so the flow near the islands was initially unperturbed and the circulation about each island is zero. The circulation thus remains zero for all time. In a discussion of flow past an offshore island it is shown in Johnson (1989) that these considerations and circulation conditions are sufficient to determine the time-varying through-gap volume fluxes and so the values of $\Psi$ at each instant on the island boundaries $\partial \mathscr{I}_{m}$. Denote these values by $\alpha_{m}$ so

$$
\Psi=\alpha_{m} \quad \text { on } \quad \partial \mathscr{I}_{m} \quad(m=1, \ldots, M) .
$$




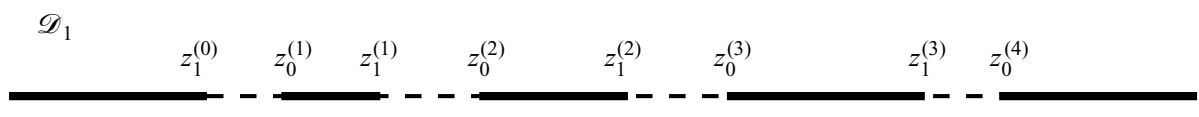

$\mathscr{D}_{2}$

FIGURE 2. Collinear gaps with islands of zero thickness. The left end of island $m$ lies at $z_{0}^{(m)}$ and the right end at $z_{1}^{(m)}$, with $z_{1}^{(0)}$ and $z_{0}^{(M)}$ at the ends of the headlands.

Introduce the streamfunction $\psi_{0}$ for an irrotational flow defined throughout $\mathscr{D}$ by

$$
\Psi=\left\{\begin{array}{lll}
\psi_{1}+\psi_{0} & \text { in } \mathscr{D}_{1} \\
\psi_{2}+\psi_{0} & \text { in } \mathscr{D}_{2}
\end{array}\right.
$$

Now $\Psi$ is continuous across gaps and $\psi_{1,2}$ vanish there, so $\psi_{0}$ is continuous across gaps. At a gap let the common normal to $\mathscr{L}_{1}$ and $\mathscr{L}_{2}$, pointing from $\mathscr{D}_{1}$ into $\mathscr{D}_{2}$ be $\boldsymbol{n}_{12}$. The normal derivative $\partial \Psi / \partial n_{12}$ is continuous across gaps, and

$$
\left[\frac{\partial}{\partial n_{12}}\left(\Psi-\psi_{0}\right)\right]=\frac{\partial \psi_{2}}{\partial n_{12}}-\frac{\partial \psi_{1}}{\partial n_{12}}
$$

where [.] denotes the jump in the enclosed quantity in moving across a gap from $\mathscr{D}_{1}$ to $\mathscr{D}_{2}$. Hence

$$
\left[\frac{\partial \psi_{0}}{\partial n_{12}}\right]=-\frac{\partial \psi_{2}}{\partial n_{12}}+\frac{\partial \psi_{1}}{\partial n_{12}}=u_{2}-u_{1}
$$

where $u_{2}$ is the tangential velocity induced at a gap by the finite-area vortices in $\mathscr{D}_{2}$, taking the gap to be a rigid wall, and $u_{1}$ the equivalent tangential velocity for the vortices in $\mathscr{D}_{1}$, with positive $u_{1}$ and $u_{2}$ corresponding to flow from left to right. The streamfunction $\psi_{0}$ is thus the irrotational flow induced in $\mathscr{D}$ by vortex sheets of strength $u_{2}-u_{1}$ lying along the gaps and specified values $\alpha_{m}$ on the $\partial \mathscr{I}_{m}$, with the $\alpha_{m}$ determined by the circulation conditions

$$
\oint \frac{\partial \psi_{0}}{\partial n} \mathrm{~d} s=-\int_{1} \frac{\partial \psi_{1}}{\partial n} \mathrm{~d} s-\int_{2} \frac{\partial \psi_{2}}{\partial n} \mathrm{~d} s \quad \text { on } \quad \partial \mathscr{I}_{m} \quad(m=1, \ldots, M),
$$

where the tangential element $\mathrm{d} s$ is taken with anti-clockwise positive, $\boldsymbol{n}$ is the outward pointing normal on $\partial \mathscr{I}_{m}$ and the integral subscripts denote that the integrations are along the sections of the island boundaries where the integrands are defined. Once $\psi_{0}$ is determined, the total streamfunction $\Psi$ is known everywhere, solving the original problem.

\subsection{Collinear gaps}

The components of the solution can be found in a number of ways depending on the shape of the full domain $\mathscr{D}$. Perhaps the most useful illustrative example is when the domain $\mathscr{D}_{1}$ is the upper half of the complex $z$-plane (i.e. $y>0$ where $z=x+\mathrm{i} y$ ) and $\mathscr{D}_{2}$ the lower half, so the islands have zero thickness and $\mathscr{L}_{1,2}$ lie along $\operatorname{Im} z=0$ as in figure 2. The streamfunction $\psi_{1}$ becomes that of a vortex distribution in the upper half-plane with a rigid planar wall along $\operatorname{Im} z=0$ and follows immediately by introducing the image vorticity distribution in the lower half-plane and solving the resulting unbounded domain problem (by, for example, contour dynamics as in §4). The streamfunction $\psi_{2}$ follows analogously. Thus the jump in the tangential velocity induced by the vortex flows on either side of $\operatorname{Im} z=0$, i.e. $\left[u_{V}\right]=u_{2}-u_{1}$, is known 
as are the vortex-induced circulations around the islands,

$$
\Gamma_{V}^{(m)}=\int_{z_{0}^{(m)}}^{z_{1}^{(m)}}\left[u_{V}\right]|\mathrm{d} z|
$$

where $z_{0}^{(m)}$ and $z_{1}^{(m)}$ are the ends of island $m$ (figure 2). It remains to obtain an expression for the irrotational field $\psi_{0}$. Denote its associated complex velocity potential by $w_{0}(z)$ and extend the complex velocity $u_{0}-\mathrm{i} v_{0}=\mathrm{d} w_{0} / \mathrm{d} z$, analytic in the upper half-plane, to the lower half-plane by

$$
\frac{\mathrm{d} w_{0}}{\mathrm{~d} z}(z)=-\frac{\overline{\mathrm{d} w_{0}}}{\mathrm{~d} z}(\bar{z}), \quad \operatorname{Im} z<0 .
$$

Then the normal velocity $v$ is continuous at gaps and the tangential velocity $u$ is odd in $y$. Since the jump in $u$ across $y=0$ is $2 u_{0}$ and $\mathrm{d} z=|\mathrm{d} z|=\mathrm{d} x$ on $y=0$, the tangential velocity condition on the irrotational flow at the gaps can be written

$$
\operatorname{Re}\left\{\mathrm{d} w_{0}\right\} /|\mathrm{d} z|=\operatorname{Re}\left\{\left(u_{0}-\mathrm{i} v_{0}\right) \mathrm{d} z\right\} /|\mathrm{d} z|=u_{0}=-\frac{1}{2}\left[u_{V}\right], \quad \text { on } y=0 .
$$

Again since $u$ is odd in $y$, the round-island circulations for irrotational flow are particularly simple, becoming for island $m$

$$
-2 \int_{z_{0}^{(m)}}^{z_{1}^{(m)}} u_{0} \mathrm{~d} x=-2 \int_{z_{0}^{(m)}}^{z_{1}^{(m)}} \operatorname{Re}\left\{\mathrm{d} w_{0} /|\mathrm{d} z|\right\}|\mathrm{d} z|=-2 \operatorname{Re}\left\{w_{0}\left(z_{1}^{(m)}\right)-w_{0}\left(z_{0}^{(m)}\right)\right\} .
$$

Thus (2.8) gives for each island

$$
\operatorname{Re}\left\{w_{0}\left(z_{1}^{(m)}\right)-w_{0}\left(z_{0}^{(m)}\right)\right\}=\frac{1}{2} \Gamma_{V}^{(m)} .
$$

These circulation conditions determine the unknown through-gap volume fluxes as the change in $\operatorname{Im} w_{0}$ across the gaps, i.e. as

$$
\alpha_{m}=\operatorname{Im}\left\{w_{0}\left(z_{0}^{(m)}\right)-w_{0}\left(z_{1}^{(m-1)}\right)\right\} .
$$

\subsection{Two gaps}

Although some progress is possible for the general geometry of figure 1 and significant progress for the linear geometry of figure 2, restricting attention to a single island $(M=2$, in figure 2) allows closed-form solutions to be derived for the flow fields and trajectories of line vortices and simple explicit expressions for evaluating the additional irrotational velocity field $\left(u_{0}, v_{0}\right)$ for vortex patches. Further, it is shown in $\S 5$ that the problem for a single island placed arbitrarily in the gap can be mapped directly to that for a symmetrically placed island. Thus attention is confined here to an island centred in the gap with results for the asymmetric case given in $\S 5$. Consider an otherwise infinite, impermeable barrier lying along $\operatorname{Im} z=0$ broken by a unit width gap at $|\operatorname{Re} z|<1$. Let the gap be interrupted by a single island at $|\operatorname{Re} z|<k$ for some $0<k<1$ (figure 3a). Then the Schwartz-Christoffel transformation given by

$$
\frac{\mathrm{d} \tau}{\mathrm{d} z}=\frac{1}{\sqrt{\left(k^{2}-z^{2}\right)\left(1-z^{2}\right)}}, \quad z=k \operatorname{sn} \tau, \quad \tau=\operatorname{sn}^{-1}(z / k),
$$

where sn denotes the elliptic function of modulus $k$, conformally maps the half-plane $\operatorname{Im} z>0$ to the rectangle $-K<\operatorname{Re} \tau<K, 0<\operatorname{Im} \tau<K^{\prime}$ in the $\tau$-plane, where

$$
K(k)=\int_{0}^{1}\left[\left(1-t^{2}\right)\left(1-k^{2} t^{2}\right)\right]^{-1 / 2} \mathrm{~d} t, \quad K^{\prime}(k)=K\left(\sqrt{1-k^{2}}\right) .
$$


(a)

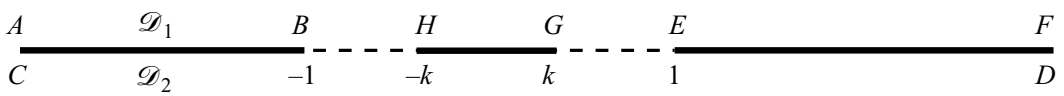

(b)

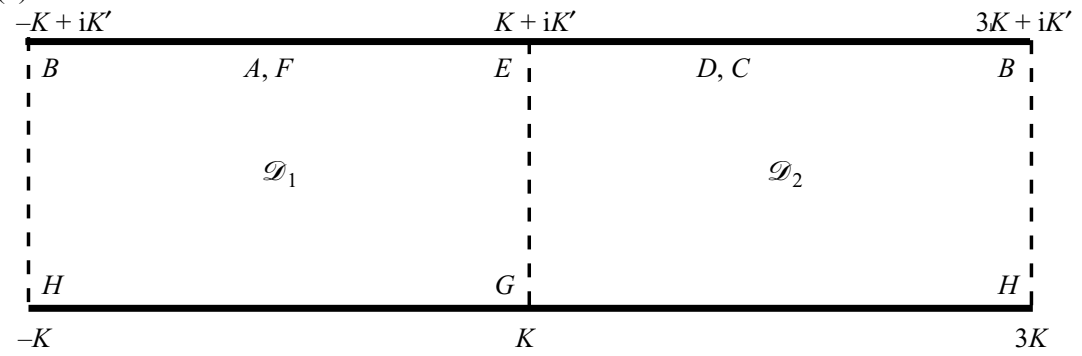

Figure 3. (a) The $z$-plane with the semi-infinite domains $\mathscr{D}_{1}$ where $\operatorname{Im} z>0$, and $\mathscr{D}_{2}$, where $\operatorname{Im} z<0$. (b) The periodic rectangle of the $\tau$-plane into which it is mapped. The points $A$ to $H$ correspond in each part.

(Here, and elsewhere, the prime does not denote a derivative.) The mapping can be interpreted as halving the straight line angles of $\pi$ at the island ends $H$ and $G$ to bring the gaps $H B$ and $G E$ vertical and simultaneously halving the angles at the barrier ends $B$ and $E$ to bring the barriers parallel so that the points $A$ and $F$ at infinity coincide at $\tau=\mathrm{i} K^{\prime}$. For $\S 3$, note that the mapping can be extended to cover the whole $z$-plane by reflecting the domain in the $z$-plane about $\operatorname{Im} z=0$, corresponding to reflecting the domain in the $\tau$-plane about $\operatorname{Re} \tau=K$. This gives the mapping of the whole $z$-plane to the periodic rectangle $0<\operatorname{Im} \tau<K^{\prime}$ in the $\tau$-plane. Figure $3(b)$ shows one real period of the $\tau$-plane rectangle where $-K<\operatorname{Re} \tau<3 K$. The line $\operatorname{Im} \tau=0$ corresponds to both sides of the island, and $\operatorname{Im} \tau=K^{\prime}$, corresponds to the semi-infinite barriers.

To determine $w_{0}$ it is sufficient to consider the rectangle $-K<\operatorname{Re} \tau<K, 0<$ $\operatorname{Im} \tau<K^{\prime}$. The velocity condition (2.10) at the gaps becomes

$$
\operatorname{Re}\left\{\mathrm{d} w_{0}\right\} /|\mathrm{d} \tau|=-\frac{1}{2}\left[u_{V}\right]|\mathrm{d} z / \mathrm{d} \tau| \quad \text { on } \quad \operatorname{Re} \tau= \pm K, \quad 0<\operatorname{Im} \tau<K^{\prime},
$$

and the flux conditions become

$$
\begin{array}{llll}
\operatorname{Im} w_{0}=\alpha & \text { on } & \operatorname{Im} \tau=0, & |\operatorname{Re} \tau|<K, \\
\operatorname{Im} w_{0}=0 \quad \text { on } & \operatorname{Im} \tau=K^{\prime}, & |\operatorname{Re} \tau|<K,
\end{array}
$$

dropping the subscript on $\alpha$ without ambiguity. The flux conditions (2.17) are simply satisfied without disturbing the tangential flow conditions (2.16) by a uniform stream in the $\operatorname{Re} \tau$-direction. Thus introduce the irrotational flow $w_{00}$ by writing

$$
w_{0}=\alpha\left(K^{\prime}-\tau\right) / K^{\prime}+w_{00} .
$$

An expression for $w_{00}$ follows directly by mapping the rectangle in the $\tau$-plane to the upper half of the annulus $\delta_{1}<|\sigma|<\delta_{2}$ in the complex $\sigma$-plane, through

$$
\sigma=\exp \left(\pi \tau / K^{\prime}\right)
$$

so $\delta_{2,1}=\mathrm{e}^{ \pm \pi K / K^{\prime}}$. Then $w_{00}$ satisfies

$$
\begin{aligned}
& \operatorname{Im} w_{00}=0 \\
& \text { on } \varphi=0, \pi, \quad \delta_{1}<|\sigma|<\delta_{2}, \\
& \operatorname{Re}\left\{\mathrm{d} w_{00}\right\} /|\mathrm{d} \sigma|=-\frac{1}{2}\left[u_{V}\right]|\mathrm{d} z / \mathrm{d} \sigma| \quad \text { on } \quad|\sigma|=\delta_{1,2}, \quad 0<\varphi<\pi,
\end{aligned}
$$


where $\varphi=\arg \sigma$. Since $w_{00}$ is analytic within the annulus, write

$$
w_{00}=\sum^{\prime} a_{n} \sigma^{n},
$$

where sums without explicit limits are taken over all integers and the prime indicates that the term corresponding to $n=0$ is omitted. This term and an otherwise allowable term in $\log \sigma$ are absent from (2.21) in order to satisfy (2.20a) as they have been absorbed into the constant $\alpha$ in (2.18). Further, since $w_{00}$ can be extended into the lower half- $\sigma$-plane so that $w_{00}(\bar{\sigma})=\overline{w_{00}(\sigma)}$, the $a_{n}$ in (2.21) can be taken as real. Then on $|\sigma|=\delta_{1,2}$, since $\mathrm{d} \sigma /|\mathrm{d} \sigma|=\mathrm{i} e^{\mathrm{i} \varphi}$ there, $(2.20 b)$ gives

$$
\operatorname{Re}\left\{\mathrm{d} w_{00}\right\} /|\mathrm{d} \sigma|=\sum^{\prime} n a_{n} \operatorname{Re}\left\{\sigma^{n-1} \mathrm{ie}^{\mathrm{i} \varphi}\right\}=\sum_{1}^{\infty} b_{n}^{(1,2)} \sin n \varphi,
$$

where

$$
b_{n}^{(1,2)}=-\left(n / \delta_{1,2}\right)\left(a_{n} \delta_{1,2}^{n}+a_{-n} \delta_{1,2}^{-n}\right), \quad n=1,2,3, \ldots
$$

Condition (2.20b) shows that the $b_{n}^{(1,2)}$ are simply the Fourier Sine coefficients in the expansion of $-\frac{1}{2}\left[u_{V}\right]|\mathrm{d} z / \mathrm{d} \sigma|$ on $|\sigma|=\delta_{1,2}, 0<\varphi<\pi$ and so are given explicitly by

$$
b_{n}^{(1,2)}=-\left(K^{\prime} / 2 \pi^{2} \delta_{1,2}\right) \int_{0}^{\pi}\left[u_{V}\right] \sqrt{\left(k^{2}-z_{1,2}^{2}\right)\left(1-z_{1,2}^{2}\right)} \sin (n \varphi) \mathrm{d} \varphi,
$$

where, from (2.14) and (2.19), $z_{2,1}=k \operatorname{sn}\left[\left(K^{\prime} / \pi\right) \log \sigma\right]=k \operatorname{sn}\left[ \pm K+\mathrm{i} K^{\prime} \varphi / \pi\right]$, giving purely real points lying within the gaps, as expected. The $b_{n}$ can be computed efficiently numerically by discrete fast Fourier transforms evaluating the integrand at points evenly spaced in $\varphi$, as in the integrations of $\S 4$ where the cost evaluating the $b_{n}$ is negligible. The coefficients in the expansion (2.21) are then given by

$$
a_{n}=\frac{\delta_{2} b_{|n|}^{(2)} \delta_{1}^{-n}-\delta_{1} b_{|n|}^{(1)} \delta_{2}^{-n}}{|n|\left(\delta_{1}^{n} \delta_{2}^{-n}-\delta_{2}^{n} \delta_{1}^{-n}\right)}, \quad n= \pm 1, \pm 2, \pm 3, \ldots
$$

It remains to obtain the through-gap volume flux $\alpha$. The island ends $z= \pm k$ correspond to $\tau= \pm K$ and $\sigma=\delta_{1,2}$. Thus

$$
\operatorname{Re}\left\{\left.w_{0}\right|_{z=k}-\left.w_{0}\right|_{z=-k}\right\}=-2 \alpha K / K^{\prime}+\sum^{\prime} a_{n}\left(\delta_{2}^{n}-\delta_{1}^{n}\right),
$$

and (2.12) gives

$$
\alpha=\frac{K^{\prime}}{2 K}\left[\frac{1}{2} \Gamma_{V}+\sum_{1}^{\infty}\left(\delta_{1} b_{n}^{(1)}+\delta_{2} b_{n}^{(2)}\right) / n\right] .
$$

This completes the solution for $w_{0}$. Vortex patch motion using this solution is described in $\S 4$ and requires the irrotational velocity field

$$
u_{0}-\mathrm{i} v_{0}=\frac{\mathrm{d} w_{0}}{\mathrm{~d} \sigma} \frac{\mathrm{d} \sigma}{\mathrm{d} \tau} \frac{\mathrm{d} \tau}{\mathrm{d} z}=\left(\pi / K^{\prime}\right)\left(\alpha \sigma / K^{\prime}+\sum^{\prime} n a_{n} \sigma^{n}\right) / \sqrt{\left(k^{2}-z^{2}\right)\left(1-z^{2}\right)},
$$

where $\sigma=\exp \left[\left(\pi / K^{\prime}\right) \operatorname{sn}^{-1}(z / k)\right]$.

\section{Line vortex flow fields and trajectories}

It is shown in Johnson \& McDonald $(2004 a, b)$ how line vortices trajectories can describe the motion of vortex patches accurately. Thus consider the motion of a set of $N$ line vortices, of strengths $\Gamma_{j}$, located at points $z=z_{j}$ and denote the complex 
velocity potential induced by these vortices at any instant by $w_{N}(z)$. Then since the flow domain is doubly path-connected the velocity field is determined only to within an arbitrary circulation around the mid-gap island or, equivalently, to within an arbitrary through-gap volume flux. As noted in $\S 2$, Kelvin's theorem requires the circulation around the island to be a constant of the motion and this constant is determined here by supposing that the vortices originated far from the origin so the flow near the island was initially unperturbed and the circulation about the island zero. A non-zero circulation corresponds simply to an additional superposed steady uniform flow in the $\operatorname{Re} \tau$-direction in the $\tau$-plane in figure 3 and can be incorporated straightforwardly, as can any imposed background irrotational flow at infinity (which corresponds to introducing sources, sinks and higher-order singularities, at the corresponding points $\tau=\mathrm{i} K^{\prime}, 2 K+\mathrm{i} K^{\prime}$ in the $\tau$-plane). In particular, if there were a net flow from $\mathscr{D}_{1}$ to $\mathscr{D}_{2}$ there would be a source at $\tau=\mathrm{i} K^{\prime}$ and an equal and opposite sink at $\tau=2 K+\mathrm{i} K^{\prime}$, corresponding to the point at infinity in the $z$-plane. Examples of flows originating at the point at infinity are given in Johnson \& McDonald (2004b) and flows with net flux from $\mathscr{D}_{1}$ to $\mathscr{D}_{2}$ in Johnson \& McDonald (2004a) and lead to similar behaviour here. As in $\S 2$, it is supposed here that there is no superposed background flow at infinity and no net flux from $\mathscr{D}_{1}$ to $\mathscr{D}_{2}$ and so the streamfunction can be taken to vanish on each semi-infinite barrier. Thus $\operatorname{Im} w_{N}$ vanishes on $\operatorname{Im} \tau=K^{\prime}$. Similarly the mid-gap island is impermeable and $\operatorname{so} \operatorname{Im} w_{N}$ is a function of time alone on $\operatorname{Im} \tau=0$, with this function $\alpha(t)$ being the instantaneous through-gap volume flux and corresponding in the $\tau$-plane to a time-varying uniform flow whose strength is a function of the instantaneous position of the vortices. Equation (2.11) shows the circulation around the island to be simply the increment in real velocity potential on going once round the island, provided the real velocity potential is continuous along the island boundary. Thus in the $\tau$-plane the zero-circulation condition becomes the continuity of $\operatorname{Re} w_{N}$ along $\operatorname{Im} \tau=0$ and

$$
\operatorname{Re} w_{N}(3 K)=\operatorname{Re} w_{N}(-K) .
$$

Let the corresponding vortex points in the $\tau$-plane be $\tau_{j}$. Then the vanishing of $\operatorname{Im} w_{N}$ on the semi-infinite barriers can be ensured by reflecting the $\tau$-plane strip about $\operatorname{Im} \tau=\mathrm{i} K^{\prime}$ and introducing image vortices of equal and opposite strengths, $\Gamma_{-j}=-\Gamma_{j}$, at the image points $\tau_{-j}=2 \mathrm{i} K^{\prime}+\overline{\tau_{j}}$. The problem becomes that of determining the motion due to $2 N$ vortices of strengths $\Gamma_{ \pm j}$ located at the points $\tau_{ \pm j}$ in the fundamental rectangle $-K<\operatorname{Re} \tau<3 K, 0<\operatorname{Im} \tau<2 K^{\prime}$, where the domain can be extended to cover the whole $\tau$-plane as doubly-periodic, with periods $4 K$ in the $\operatorname{Re} \tau$-direction and $2 \mathrm{i} K^{\prime}$ in the $\operatorname{Im} \tau$-direction. Associated with each vortex is a cut in the $\tau$-plane to give a single-valued definition of $w_{N}$. Provided none of these cuts crosses $\operatorname{Im} \tau=0, \operatorname{Re} w_{N}$ will be continuous along $\operatorname{Im} \tau=0$ and so the circulation condition (3.1) will be satisfied provided $w_{N}(\tau)$ has real period $4 K$. This is achieved straightforwardly by taking the cuts to join each vortex to its image in the fundamental rectangle as in figure 4 . The choice of the cuts and requirement of periodicity determine the through-gap volume flux, $\alpha$, uniquely.

Since the Weierstrass $\zeta$-function, $\zeta(z)=\zeta\left(z ; 2 K\right.$, i $\left.K^{\prime}\right)$ (Whittaker \& Watson 1927, Chap. 20) is analytic everywhere except for simple poles of residue unity on the lattice with periods $4 K$ and $2 \mathrm{i} K^{\prime}$ centred on the origin, the complex velocity induced by the vortices in the $\tau$-plane can be written

$$
u-\mathrm{i} v=\frac{\mathrm{d} \bar{\tau}}{\mathrm{d} t}=\frac{1}{2 \pi \mathrm{i}} \sum_{-N}^{N} \Gamma_{j} \zeta\left(\tau-\tau_{j}\right)+C,
$$




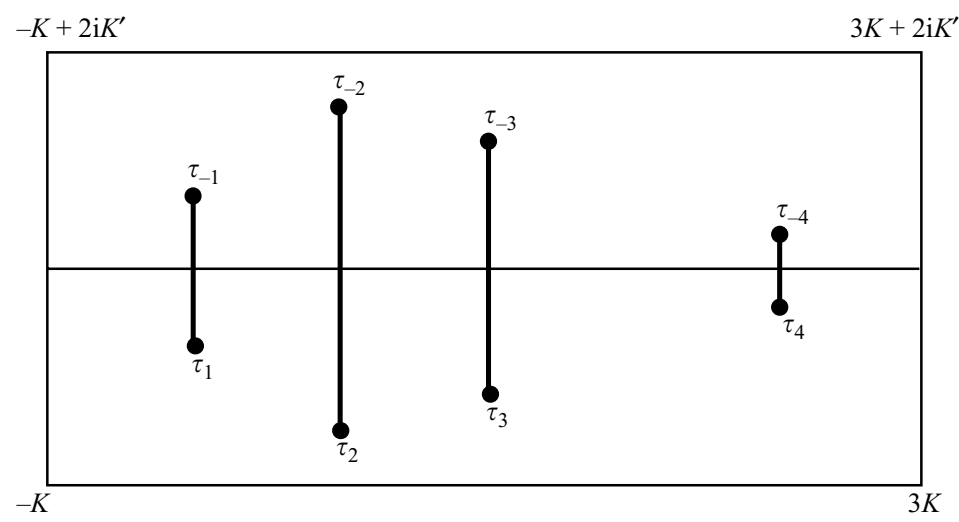

FIGURE 4 . The fundamental rectangle in the $\tau$-plane showing the image vortices, $\tau_{-j}$, joined to the mapped $z$-plane vortices, $\tau_{j}$, by cuts crossing $\operatorname{Im} \tau=\mathrm{i} K^{\prime}$, corresponding to the semi-infinite barriers. No cuts cross $\operatorname{Im} \tau=0$ and so $\operatorname{Re} w_{N}$ is continuous along this line, which corresponds to the island boundary. Hence provided $w_{N}$ has period $4 K$ the round-island circulation vanishes.

where the prime denotes that there is no term corresponding to $j=0$. By Liouville's theorem the sum in (3.2) can differ by at most a constant, $C$ here, from the required velocity field. The constant $C$, independent of $\tau$ but possibly depending on $\tau_{1}, \ldots, \tau_{N}$, gives a uniform flow in the $\tau$-plane and thus is proportional to the through-gap volume flux in the $z$-plane. The $\zeta$-function is quasi-periodic and related to Jacobi's $\vartheta_{1}$-function through

$$
\zeta(z)=\left(\eta_{1} / 4 K\right) z+\frac{\mathrm{d}}{\mathrm{d} z} \log \vartheta_{1}(\pi z / 4 K),
$$

where $\eta_{1}=2 \zeta(2 K)$ and the nome $\exp \left(-\pi K^{\prime} / 2 K\right) \dagger$ of $\vartheta_{1}$ is suppressed here and below. Integrating (3.2) with respect to $\tau$ then gives the complex potential for the induced flow, omitting the arbitrary additive constant, as

$$
w_{N}=\frac{1}{2 \pi \mathrm{i}} \sum_{-N}^{N} \Gamma_{j}\left[\log \left(\vartheta_{1}\left[\pi\left(\tau-\tau_{j}\right) / 4 K\right]\right)+\left(\eta_{1} / 8 K\right)\left(\tau-\tau_{j}\right)^{2}\right]+C \tau .
$$

Provided the sum is evaluated with the pairing noted above, and shown in figure 4, $\operatorname{Re} w_{N}$ is continuous along $\operatorname{Im} \tau=0$. Since $\vartheta_{1}(\tau+1)=-\vartheta_{1}(\tau)$ for all $\tau$, the logarithmic terms in (3.4) have period $4 K$ as required. Moreover the coefficient of $\tau^{2}$ vanishes as the total circulation is zero. Hence the zero circulation condition (3.1) requires the coefficient of $\tau$ to vanish. Thus

$$
C\left(\tau_{1}, \tau_{2}, \ldots, \tau_{N}\right)=\left(\eta_{1} / 8 \pi \mathrm{i} K\right) \sum_{-N}^{N} \Gamma_{j} \tau_{j}=\left(\eta_{1} / 4 \pi K\right) \sum_{1}^{N} \Gamma_{j}\left(\operatorname{Im} \tau_{j}-K^{\prime}\right),
$$

which is real, giving a velocity parallel to the impermeable barriers at $\operatorname{Im} \tau=0, K^{\prime}$ as expected. This completes the determination of the complex velocity (3.2) and shows that, to within an arbitrary additive constant, the complex velocity potential of the

$\dagger$ Here, as usual, the nome is formed from the ratio of the periods of an elliptic function but is the square root of the usual nome as the real period has been doubled to $4 K$. 

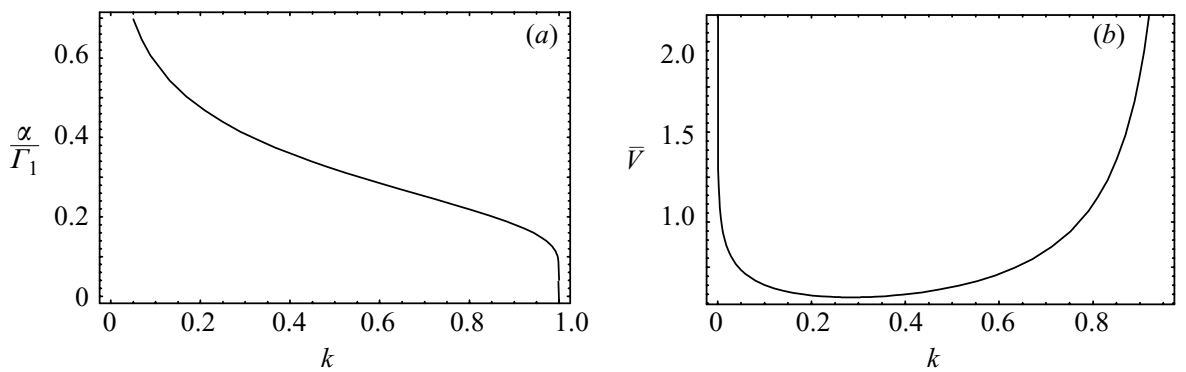

Figure 5. (a) The through-gap flux as a function of the island half-width, $k$, for a line vortex against the island (on $\operatorname{Im} \tau=0$ ), normalized on the vortex strength. (b) The corresponding average through-gap velocity $\bar{V}$ as a function of $k$. When $k=0$ the island is absent and the flux is dominated by the infinite velocity at the centre of the line vortex. For $k \rightarrow 1$ the island fills the entire gap.

induced field has the particularly simple form $\dagger$

$$
w_{N}=\frac{1}{2 \pi \mathrm{i}} \sum_{-N}^{N}{ }^{\prime} \Gamma_{j} \log \left(\vartheta_{1}\left[\pi\left(\tau-\tau_{j}\right) / 4 K\right]\right) .
$$

Since $\operatorname{Im} w_{N}\left(\mathrm{i} K^{\prime}\right)=0$, the through-gap volume flux is given, using $\vartheta_{1}\left(z+\mathrm{i} \pi K^{\prime} / 2 K\right)=$ $-\vartheta_{1}(z) \exp \left(\pi K^{\prime} / 2 K-2 \mathrm{i} z\right)$, by

$$
\alpha\left(\tau_{1}, \tau_{2}, \ldots, \tau_{N}\right)=\operatorname{Im} w_{N}(0)=(1 / 4 K) \sum_{1}^{N} \Gamma_{j}\left(K^{\prime}-\operatorname{Im} \tau_{j}\right)=-\pi C / \eta_{1} .
$$

As the problem in this domain is invariant under translations in the $\operatorname{Re} \tau$-direction, both $\alpha$ and $C$ are independent of the $\operatorname{Re} \tau_{j}$ and, as the flow fields due to individual vortices are proportional to their strengths and additive, $\alpha$ and $C$ consist of sums of individual vortex terms proportional to the $\Gamma_{j}$. They are proportional to the vortex impulse in the $\operatorname{Re} \tau$-direction, a constant of the motion in the $\tau$-plane. A positive vortex $\left(\Gamma_{j}>0\right)$ contributes to a flux in the $-\operatorname{Re} \tau$-direction, corresponding to an anti-clockwise volume flux through the gaps, an amount decreasing linearly with $\tau_{j}$ from a maximum value of $\Gamma_{j} K^{\prime} / 4 K$ when the vortex is near the island to zero when the vortex lies near the barriers. Figure 5(a) gives the through-gap volume flux, normalized on the vortex strength, for a vortex against the island as a function of the island half-length $k$. The flux becomes logarithmically infinite as $k \rightarrow 0\left(K^{\prime} / 4 K \rightarrow(1 / 4 \pi) \log \left(16 / k^{2}\right)\right)$ due to the infinite velocities associated with the point vortex. For a vortex patch, induced velocities are finite and so the flux remains finite and simply becomes the flux in the absence of an island as $k \rightarrow 0$. The flux vanishes as $k \rightarrow 1$. However it decreases only slowly $\left(K^{\prime} / 4 K \rightarrow \pi / 4 \log \left[16 /\left(1-k^{2}\right)\right]\right)$ and so the average normalized through-gap velocity $\left(\bar{V}=K^{\prime} / 4(1-k) K\right)$ in figure $5(b)$ becomes infinite as $k \rightarrow 1$. This is due entirely to the circulation condition and the leading-order behaviour is unaltered if the line vortex is replaced by a vortex patch. Figure 6 shows instantaneous streamlines for $k=0.8$ for a vortex at $z=0.4+0.2 \mathrm{i}$. The flow is periodic in $\operatorname{Re} \tau$-direction and the impermeable boundaries $\operatorname{Im} \tau=0, \mathrm{i} K^{\prime}$ are streamlines. The circulation condition on the island requires a uniform flow (to Abramowitz \& Stegun 1972) give simple rapidly convergent series. 
(a)

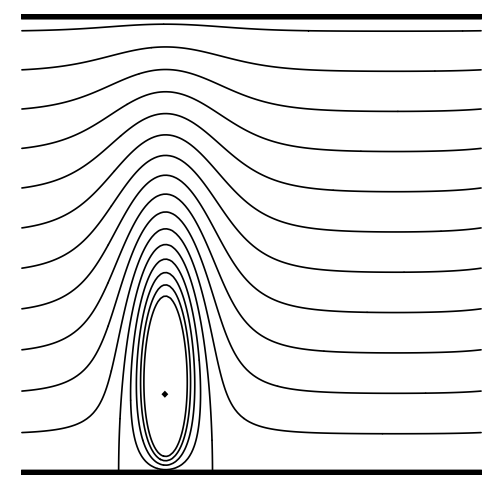

(b)

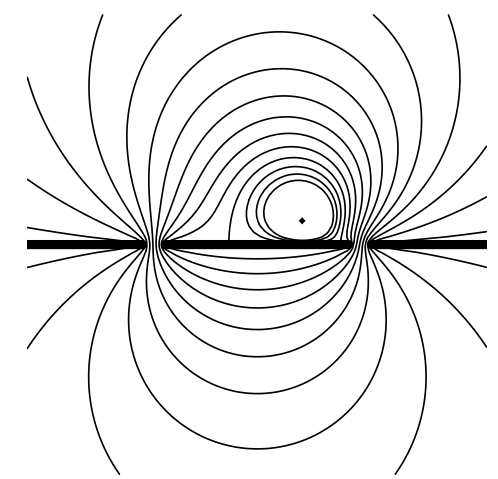

FIGURE 6. Instantaneous streamlines for a vortex, shown by the small diamond, at $z=0.4+0.2 \mathrm{i}$ for an island lying in $|x| \leqslant 0.8$ in the in gap $|x| \leqslant 1$. (a) In the $\tau$-plane. The flow is periodic in the $\operatorname{Re} \tau$-direction and the impermeable boundaries $\operatorname{Im} \tau=0, \mathrm{i} K^{\prime}$ are streamlines. The circulation condition on the island requires a uniform flow (to the left for a positive vortex) sufficiently strong to introduce two stagnation points on the island. (b) In the $z$-plane. Again the circulation condition introduces two stagnation points on the island. The circulation condition induces a rapid flow through each of the gaps.

the left for a positive vortex) sufficiently strong to introduce two stagnation points on the island. There are equal and opposite rapid flows through the gaps each of which behaves as a localized sink in one half-plane and source in the other.

The complex velocity of vortex $m$ moving in the $\tau$-plane under the influence of the other vortices is given by

$$
u_{m}-\mathrm{i} v_{m}=\frac{\mathrm{d} \overline{\tau_{m}}}{\mathrm{~d} t}=\lim _{\tau \rightarrow \tau_{m}}\left(\frac{\mathrm{d} w_{N}}{\mathrm{~d} \tau}-\frac{\Gamma_{m}}{2 \pi \mathrm{i}\left(\tau-\tau_{m}\right)}\right) .
$$

Since $\zeta(z)-1 / z$ vanishes at $z=0$, this expression is precisely (3.2) with the term $j=m$ omitted,

$$
u_{m}-\mathrm{i} v_{m}=\frac{1}{2 \pi \mathrm{i}} \sum_{-N}^{N}{ }^{\prime \prime} \Gamma_{j} \zeta\left(\tau_{m}-\tau_{j}\right)+\left(\eta_{1} / 4 \pi K\right) \sum_{1}^{N} \Gamma_{j}\left(\operatorname{Im} \tau_{j}-K^{\prime}\right),
$$

where the double prime denotes the absence of terms for $j=0, m$. Alternatively,

$$
\begin{aligned}
u_{m}-\mathrm{i} v_{m}=\left(\Gamma_{m} / 2 \pi \mathrm{i}\right)\{-\zeta & {\left.\left[2 \mathrm{i}\left(\operatorname{Im} \tau_{m}-K^{\prime}\right)\right]+\left(\eta_{1} / 4 K\right)\left[2 \mathrm{i}\left(\operatorname{Im} \tau_{m}-K^{\prime}\right)\right]\right\} } \\
+ & \frac{1}{2 \pi \mathrm{i}} \frac{\mathrm{d}}{\mathrm{d} \tau_{m}} \sum_{-N}^{N}{ }^{\prime \prime \prime} \Gamma_{j} \log \left(\vartheta_{1}\left[\pi\left(\tau_{m}-\tau_{j}\right) / 4 K\right]\right),
\end{aligned}
$$

where the triple prime denotes the absence of terms for $j=0, \pm m$, and so can be derived from the Hamiltonian

$$
\begin{aligned}
& H^{(\tau)}\left(\tau_{1}, \tau_{2}, \ldots, \tau_{N}\right)=\frac{1}{4 \pi} \sum_{1}^{N} \Gamma_{j}^{2} \log \left|\vartheta_{1}\left[2 \pi \mathrm{i}\left(\operatorname{Im} \tau_{j}-K^{\prime}\right) / 4 K\right]\right| \\
& +\frac{1}{2 \pi} \sum_{j=1}^{N} \sum_{m=j+1}^{N} \Gamma_{j} \Gamma_{m} \log \left|\vartheta_{1}\left[\pi\left(\tau_{k}-\overline{\tau_{m}}-2 \mathrm{i} K^{\prime}\right) / 4 K\right] / \vartheta_{1}\left[\pi\left(\tau_{m}-\tau_{j}\right) / 4 K\right]\right| .
\end{aligned}
$$


The Hamiltonian is a constant of the motion in the $\tau$-plane, proportional to the energy of the vortex system. As the problem is invariant under translations in the $\operatorname{Re} \tau$-direction, the impulse in the $\operatorname{Re} \tau$-direction, $\sum_{1}^{N} \Gamma_{j} \operatorname{Im} \tau_{j}$, is a second constant of the motion and thus $\alpha$ (and so $C$ ) is a constant of the motion in the $\tau$-plane.

To return to the original $z$-plane, note that the fundamental rectangle in the $\tau$ plane corresponds to a two-sheeted Riemann surface in $z$. The lower half of the fundamental rectangle in the $\tau$-plane corresponds to the full $z$-plane flow domain cut along the semi-infinite barriers and along the mid-gap island. The upper half of the fundamental rectangle, containing the image vortices, corresponds to the other full $z$-plane sheet, the image sheet, joined to the flow domain along the cuts. Thus an image vortex at $\tau_{-j}=2 \mathrm{i} K^{\prime}+\overline{\tau_{j}}$ is mapped back to an image vortex at $z_{-j}=\overline{z_{j}}$, but on the second, image, Riemann sheet. The Hamiltonian for the $z$-plane motion is related to that in the $\tau$ through

$$
H^{(z)}\left(z_{1}, z_{2}, \ldots, z_{N}\right)=H^{(\tau)}\left(\tau_{1}, \tau_{2}, \ldots, \tau_{N}\right)+\frac{1}{4 \pi} \sum_{j=1}^{N} \Gamma_{j}^{2} \log |\mathrm{d} z / \mathrm{d} \tau|_{\tau_{j}}
$$

(Routh 1881; Lin 1941; discussed in Saffman 1992). Thus interpreting $z_{-j}$ so that $\mathrm{i} K^{\prime}<\operatorname{Imsn}^{-1}\left(z_{-j} / k\right)<2 \mathrm{i} K^{\prime}$ in the upper half of the fundamental rectangle, and noting that the modulus of the transformation (2.14) is both independent of the sheet on which $z_{j}$ lies and the same for $z_{j}$ and $\overline{z_{j}}$, gives the Hamiltonian in the $z$-plane as

$$
H^{(z)}\left(z_{1}, z_{2}, \ldots, z_{N}\right)=H^{(\tau)}\left(\tau_{1}, \tau_{2}, \ldots, \tau_{N}\right)+\frac{1}{8 \pi} \sum_{j=1}^{N} \Gamma_{j}^{2} \log \left|\left(k^{2}-z_{j}^{2}\right)\left(1-z_{j}^{2}\right)\right|,
$$

where $\tau_{j}=\operatorname{sn}^{-1}\left(z_{j} / k\right)$ for each $j$. For a single vortex (3.13) becomes

$$
H^{(z)}\left(z_{1}\right)=\left(\Gamma_{1}^{2} / 4 \pi\right) \log \left|\left(k^{2}-z_{1}^{2}\right)^{1 / 2}\left(1-z_{1}^{2}\right)^{1 / 2} \vartheta_{1}\left[\mathrm{i} \pi\left(\operatorname{Im~sn}^{-1}\left(z_{1} / k\right)-K^{\prime}\right) / 2 K\right]\right| .
$$

Again the Hamiltonian is a constant of the motion. However no component of the impulse is conserved either in the $z$-domain or for the mapped trajectories in the $\tau$-plane and so the through-gap volume flux $\alpha$ (and also $C$ ) varies as the vortices move.

Figure 7 shows contours of the Hamiltonian (3.13) for three island lengths. The Hamiltonian has two saddle points of equal height (symmetrically placed about the origin) within the gaps. The separatrices originating at these saddle points divide trajectories into three types: vortices sufficiently close to a headland pass through the first gap they encounter; vortices sufficiently close to the island orbit the island driven by the image vorticity (of zero total circulation) they induce in the island; and vortices further away pass by the gap. These last vortices are nevertheless strongly affected by the gaps. As a vortex approaches the gap it begins to turn in as though the island were not there and the gap occupied the whole region $|\operatorname{Re} z|<1$, as in the single-gap trajectories of Johnson \& McDonald (2004a). Sufficiently close to the island the induced image vorticity drives the through-gap flux of figure $6(b)$ and the vortex behaves as a vortex passing a single gap with flow inwards through the gap (Johnson \& McDonald 2004a): the vortex passes the gap but remains closer to the wall on the far side. The vortex propagates along the island to the second gap where it behaves as a vortex passing a single gap with outflow. It passes the gap but moves outward to be further from the wall on the far side. As the island is symmetrically placed in the gap here vortex trajectories are symmetric about $\operatorname{Im} z=0$. (Differences for asymmetric gaps, when the saddle points of the Hamiltonian have different heights, are noted in 
(a)

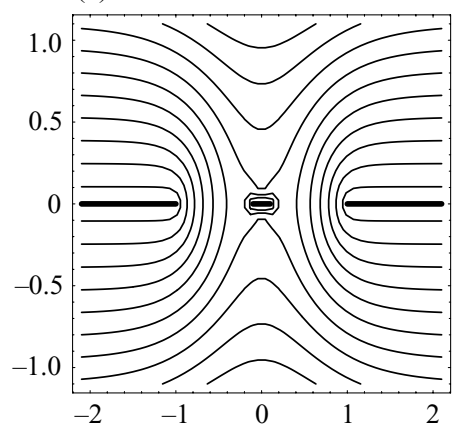

(c)

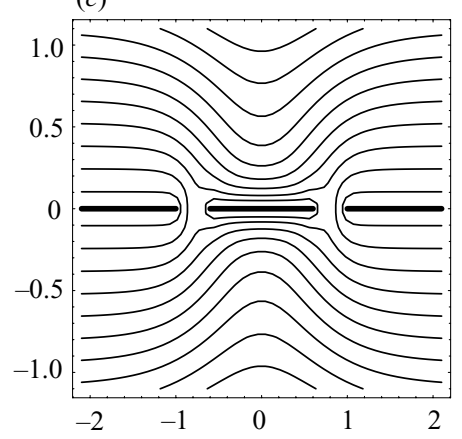

(e)

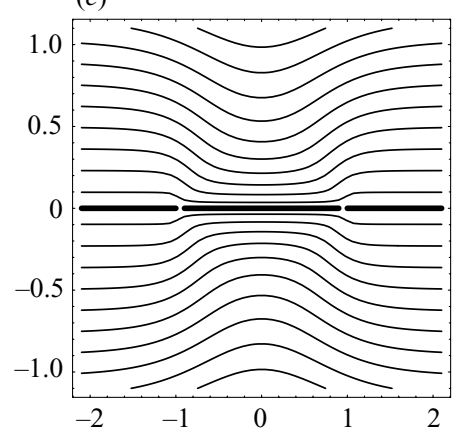

(b)

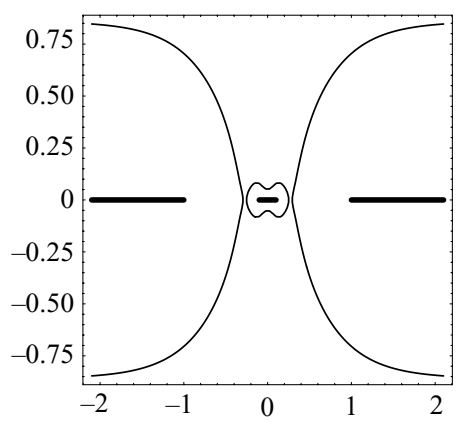

(d)
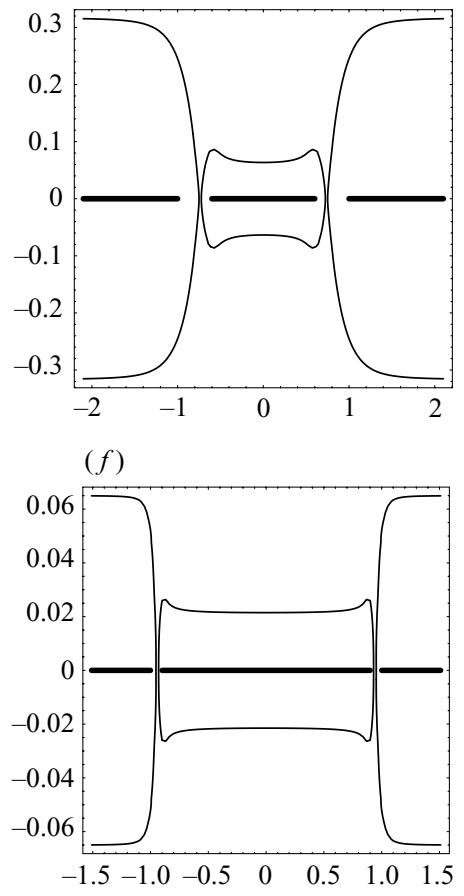

FIGURE 7. Vortex trajectories (left) and separatrices (right) for various island lengths. $(a, b) k=0.1$. $(c, d) k=0.6$. $(e, f) k=0.9$.

$\S 5$.) Since the induced velocities at the gaps are greatest for the smallest gaps, this effect is most noticeable for the largest islands. Thus the trajectories for $k=0.9$ in figure 7(e,f) dip in markedly even for vortices starting (at $\operatorname{Re} z= \pm \infty,|\operatorname{Im} z|>1$ ) more than one gap width away from the headlands. Trajectories starting there for the small island $(k=0.1)$ of figure $7(a, b)$ are relatively unperturbed. The fraction occupied by the island does not have to be extreme and figure $7(c, d)$ shows the strong influence of the island when $k=0.6$, an island occupying $60 \%$ of the total gap width.

\section{Vortex patch dynamics}

Contour dynamics (Deem \& Zabusky 1978; Zabusky et al. 1979; Saffman 1992) computes the motion of finite-area constant-vorticity patches in an unbounded domain 
highly accurately by following only the boundaries of the patches. Integrations can be continued to large times with practically no loss of accuracy provided boundary contours are allowed to break and join through contour surgery (Dritschel 1988). The algorithm requires the Green's function for the underlying Poisson equation to be of a particularly simple form (see Johnson \& Clarke 1998 for a general discussion and some examples). It can however be extended to more general domains by using the contour dynamics algorithm to compute the vortical part of the flow field using the simple unbounded-domain Green's function and then, at each time step, constructing an irrotational flow to satisfy the boundary conditions on any solid boundaries (e.g. an elliptical island in Johnson \& Clarke 1998). It is thus directly applicable to the ansatz described in $\S 2$.

The method follows that described in Johnson \& McDonald (2004a). At each timestep the velocity field $-\boldsymbol{k} \times \nabla \psi_{1}$ from vortex patches in $\operatorname{Im} z>0$ with a solid wall along $\operatorname{Im} z=0$ is computed, satisfying impermeability at the wall by introducing image vortex patches in $\operatorname{Im} z<0$. Vortices straddling $\operatorname{Im} z=0$ are truncated. The velocity field is calculated, as usual, at all points defining the vortex contours in $\operatorname{Im} z>0$ and additionally the tangential velocity $u_{1}$ is computed for the discrete points $z_{j}^{(1,2)}=k \operatorname{sn}\left[ \pm K+\mathrm{i} j K^{\prime} / N_{F} \pi\right]\left(j=1, \ldots, N_{F}\right)$ inside the gaps and for $N_{I}$ points evenly spaced along the island. This is repeated for vortex patches in the $\operatorname{Im} z<0$ to obtain the velocity field $-\boldsymbol{k} \times \nabla \psi_{2}$ for contours in $\operatorname{Im} z<0$ and the tangential velocity $u_{2}$ at the gap points $z_{j}^{(1,2)}$ and the island points. Integrating the tangential velocity jump along the island gives the patch-induced circulation $\Gamma_{V}$ of (2.8). Substituting the tangential velocity jump in (2.24), truncating the infinite sum and numerically inverting the Fourier series gives the coefficients $b_{n}, n=1, \ldots, N_{F}$ from which (2.27) and (2.25) give $\alpha$ and the $a_{n}$. Evaluating the series (2.28) gives the required additional irrotational velocity field at each point on a patch boundary. In the examples here the vorticity in each patch is taken to be unity. Changing this value simply alters the timescale of the motion, with higher vorticity giving faster propagation. Patches are advected using a fourth-order Runge-Kutta method with a timestep of $\Delta t=0.05$ unless the vortex interacts strongly with the island in which case the timestep is reduced to $\Delta t=0.01$ to accommodate the large velocities near the island. Points are redistributed along the contour after each timestep based on the curvature of the contour. The algorithm was tested by comparing trajectories of patches with initially small radii with those obtained in $\S 3$ for line vortices. In the integrations described below the initial vorticity distribution is taken always to be a single circular vortex patch and computational points are placed along the contour with resolution (i.e. spacing along the contour) of at most a tenth the initial radius. $N_{F}=100$ points are distributed across each gap and $N_{I}=100$ points along the island.

As for a line vortex, once the circulation is absorbed into the timescale the evolution of a vortex patch is determined by the initial position of its centroid. In addition, the vortex patches here have an extra parameter: their initial radius (if the patches were not initially circular more parameters would be needed). It is shown in Johnson \& McDonald (2004b) that a vortex patch centroid is constrained to follow the trajectory of a line vortex with the same circulation even in the presence of strong boundary effects provided solely that the patch remains circular. In fact the patch centroid follows the trajectory at the same speed as the line vortex as noted briefly in the Appendix. A typical evolution for a vortex that passes through the first gap is shown in figure 8 . The island occupies $40 \%$ of the full gap width $(k=0.4)$, and the vortex has an initial radius of 0.1 , starting with its centroid 0.4 from the wall. This puts it inside the separatrix for line vortices to pass through the gap and, indeed, the 

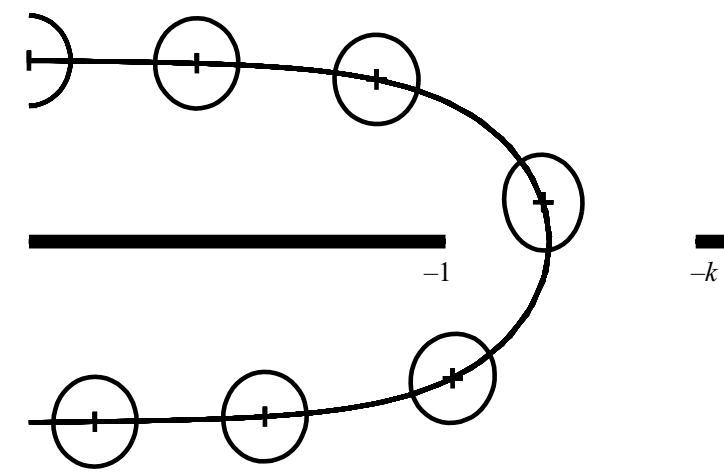

FIGURE 8 . The motion of a vortex with initial radius 0.1 and centroid position $(-2,0.4)$. Snapshots of the vortex are taken at $t=0,10,20,30,40,50$ and 60 . The patch centroid is shown by a cross. The solid line gives the trajectory of a line vortex starting at the same place. Here $k=0.4$ so the island fills $40 \%$ of the gap.

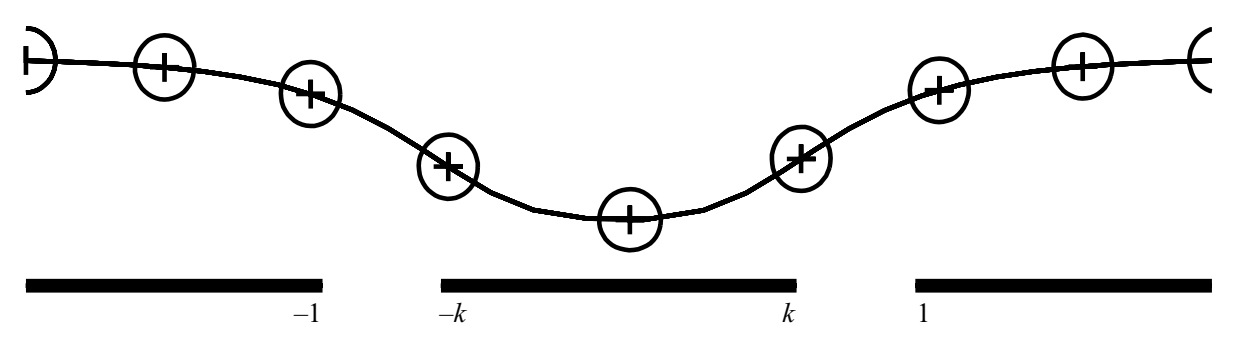

FIgURE 9. As in figure 8 but for a vortex starting at $(-2,0.7)$ and snapshots at $t=0,20,40$, $60,80,100,120,140$ and 160.

vortex patch passes through the gap, following the equivalent line vortex trajectory extremely closely. Figure 9 shows the same case but with the vortex centroid initially 0.7 from the wall. This puts it outside the separatrix and the vortex passes by the gap, dipping in towards the island and travelling along it before moving out to its initial distance from the wall on passing the second gap. Again the patch follows the equivalent line vortex trajectory extremely closely. Figure 10 shows how accurately the line vortex separatrix can predict the dramatically different behaviour of the vortex patches for small changes of initial conditions. It shows the centroid paths of a single patch from two integrations where the initial displacement of the patch from the wall changes by less than $1.5 \%$. Still the patch inside the separatrix passes through the gap and that outside passes by the gap. Since trajectories passing by the gap can dip in closely towards the central island sufficiently large vortices can be carried inwards to collide with the island. Figure 11 shows a vortex of radius 0.3 striking the island. The equivalent line vortex path passes by the gap and initially the patch centroid follows this closely. However on striking the island the vortex is sufficiently distorted from circular to temporarily break the constraint of following the line vortex path. The patch passes through the gap and returns to an approximately circular shape. It is thus again constrained to travel along the path of an equivalent line vortex of the same energy and it accurately follows this path on the far side of the gap. Figures 12 and 13 show large patches passing close to small gaps. The around-island volume flux forces much of the patches to squeeze through the first gap so that the eddies on the far side of the gap have diameters significantly larger than the gap width. 


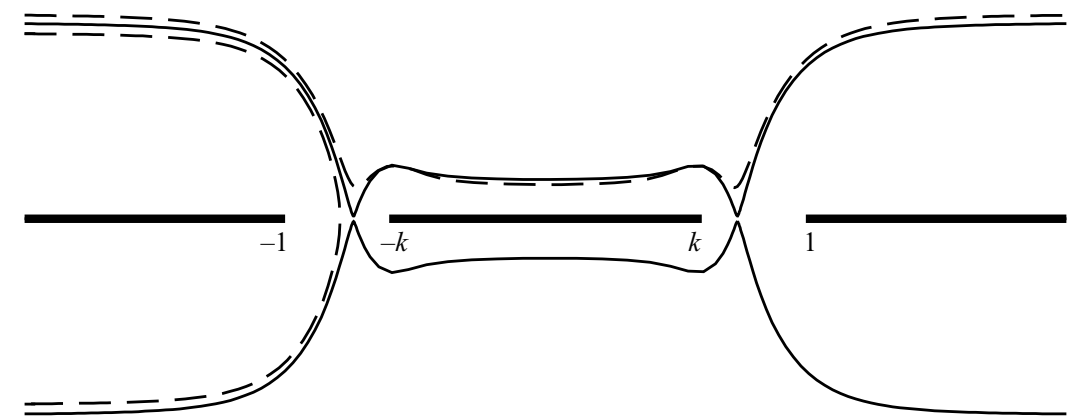

Figure 10. The paths of vortex patch centroids (dashed lines) relative to the line vortex separatrix (solid line) for $k=0.6$ so the island occupies $60 \%$ of the gap. The patches each have initial radius 0.1 . One patch centroid starts at $(-2,0.33)$, further from the headland than the separatrix, and the patch jumps both gaps. The other patch starts at $(-2,0.3)$, inside the separatrix, and passes through the first gap. Although the patches occupy half the gap width their paths are closely predicted by equivalent line vortices.

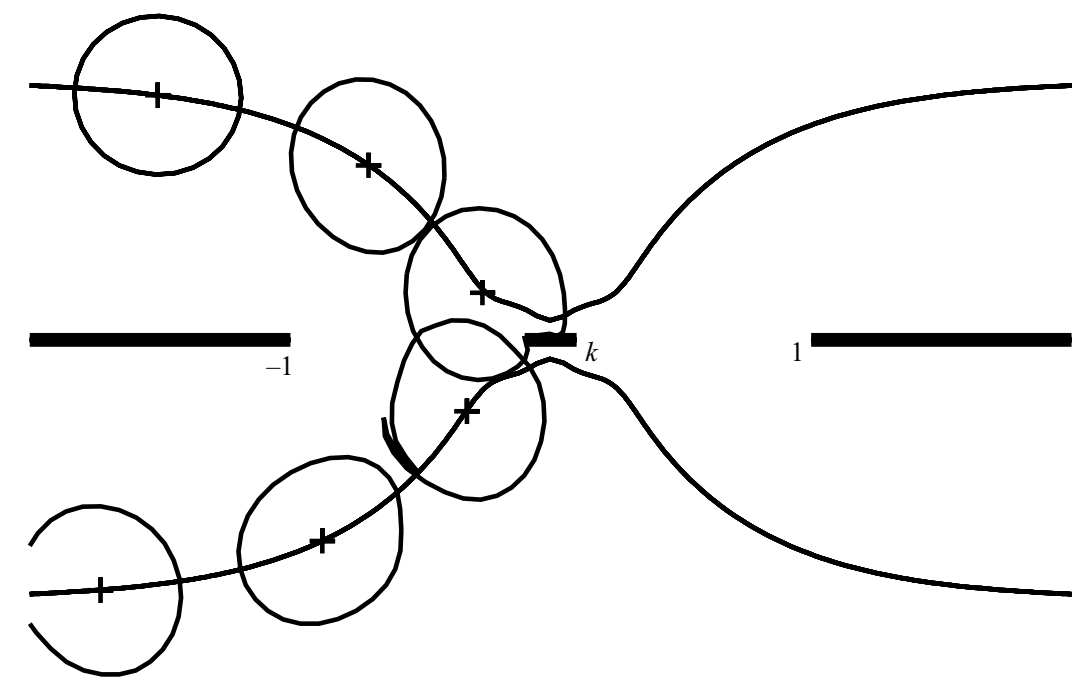

Figure 11. As for figure 8 but for a smaller island with $k=0.1$ and a larger vortex, of initial radius 0.3 , starting $(-2,0.9)$. The snapshots are at $t=3,8,13,18,23$ and 28 . The patch strikes the island and is sufficiently distorted from circular for its centroid to leave the line vortex path before the patch resymmetrises and the centroid returns to a different Hamiltonian isoline still, however, corresponding to the initial patch energy.

\section{Discussion}

This paper has described the motion of point vortices and vortex patches near multiple gaps. Fully nonlinear explicit analytical expressions for the paths of single line vortices near a pair of gaps have been derived by considering the Hamiltonian for the flow. It has been shown that the constraint that the circulation around an island within a gap remains constant throughout the motion means that strong flows are driven through narrow gaps at either end of the island. Finite-area vortex patches are shown to follow the line vortex trajectories provided the vortices remain close to circular. However the strong flows near narrow gaps can tear the patches apart, in general forcing more of the patch through the gap than might otherwise be expected: 

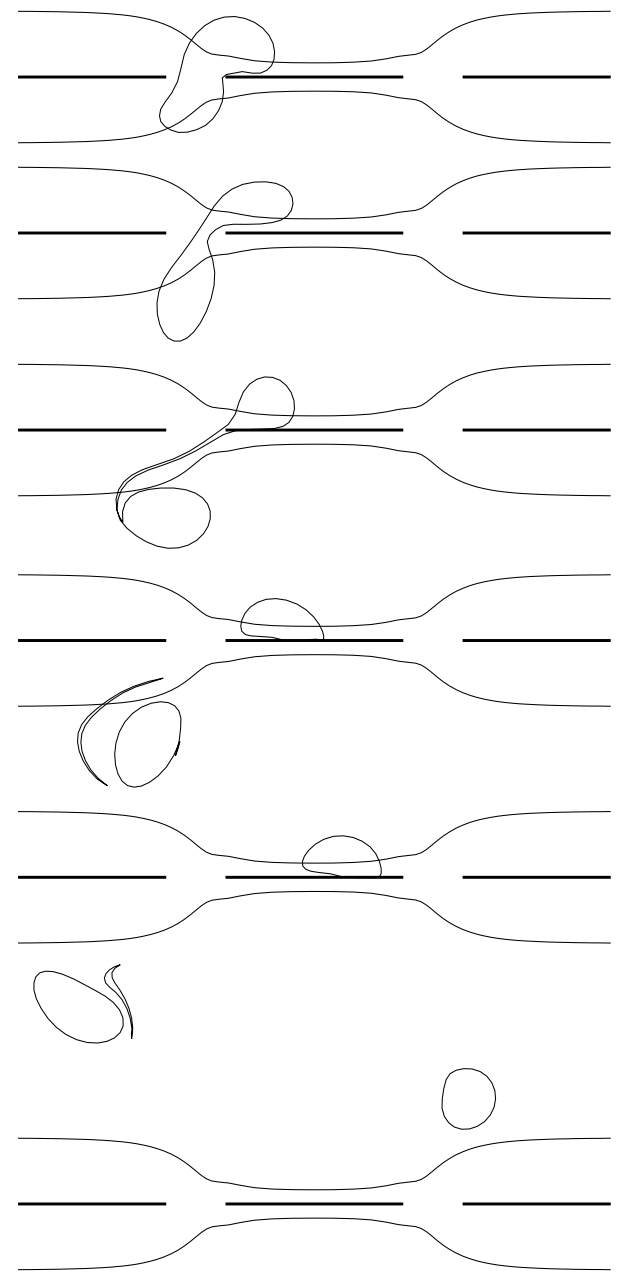

Figure 12. As for figure 8 but with $k=0.6$ and a patch of radius 0.3 starting from $(-2,0.4)$. The snapshots are at $t=4,5,6,7,10$ and 20. The round-island volume flux forces the larger part of the patch to pass through the first gap even though the patch is $50 \%$ wider than the gap.

eddies on the far side of gaps, as in figures 12 and 13 have diameters significantly larger than the gap width. This leads to the hypothesis that because of the circulation constraint island chains may be only weak barriers to eddy motion. A testing of this hypothesis in a model with more complex physics and geometry would appear to be of great interest.

The underlying conformal invariance of the irrotational problem means that the results here for line vortex trajectories extend immediately to other geometries and in many cases so do the integrations for finite-area vortices. An island in the $\hat{z}$-plane with end points $\hat{z}=x_{0}$ and $\hat{z}=x_{1}$ (with $-1<x_{0}<x_{1}<1$ ) lying asymmetrically within a gap of width 2 is mapped back to the symmetric problem in the $z$-plane by the bilinear transformation

$$
z=G^{-1}(\hat{z})=(-\alpha \hat{z}+1) /(\hat{z}-\alpha)
$$




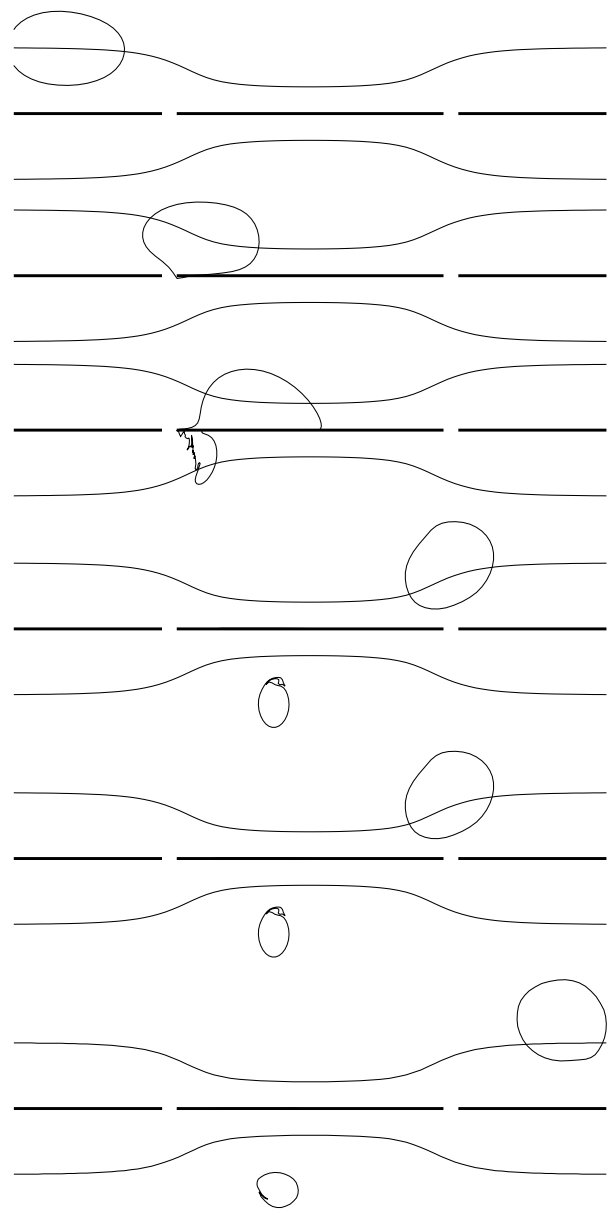

FIGURE 13. As in figure 12 but for an island occupying $90 \%$ of the gap $(k=0.9)$. The snapshots are at $t=1,3.5,4.5,7,9$ and 12. The patch is six times wider than the gap yet a significant amount of the patch (with diameter more than double the gap width) is forced through the first gap.

which leaves the semi-infinite barriers and gap of width 2 unchanged but moves the island to lie symmetrically within the gap along $|\operatorname{Re} z| \leqslant k<1$. Here $\alpha=\mathrm{e}^{\beta}$ and $k=\mathrm{e}^{\gamma}$ where

$$
\beta=\cosh ^{-1}\left[\left(x_{0} x_{1}+1\right) /\left(x_{0}+x_{1}\right)\right], \quad \gamma=-\cosh ^{-1}\left[\left(x_{0} x_{1}-1\right) /\left(x_{0}-x_{1}\right)\right] .
$$

The methods above for symmetrically located islands apply directly to give point vortex and vortex patch trajectories. For example, since $G^{\prime}(z)=(\hat{z}-\alpha)^{2} /\left(\alpha^{2}-1\right)$, the Hamiltonian for a single point vortex follows as

$$
H=\left(\Gamma^{2} / 4 \pi\right) \log \left|\left(k^{2}-z^{2}\right)^{1 / 2}\left(1-z^{2}\right)^{1 / 2} \theta_{1}\left[\operatorname{i} \pi\left(\operatorname{Im} \tau-K^{\prime}\right) /(2 K)\right](\hat{z}-\alpha)^{2}\right| .
$$

Figure 14 shows a vortex jumping a narrow gap before passing through a second, wider gap.

The headlands have so far been taken to be thin barriers but simple transformations allow these to have widths that increase away from the gap. An extreme example of 


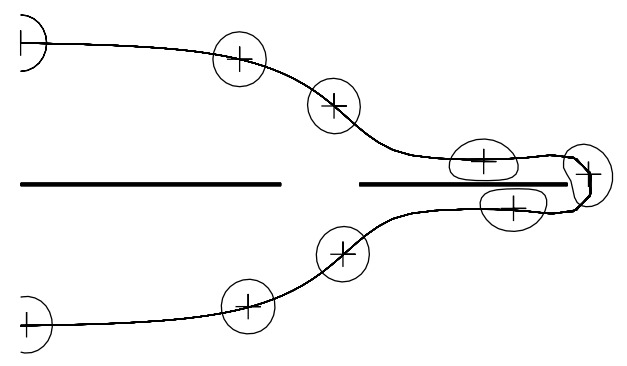

FIGURE 14. An island with ends at $x_{0}=-0.7$ and $x_{1}=0.1$ lying off-centre in the gap $|\operatorname{Re} z|<1$. The vortex jumps the first gap but passes through the second, wider gap.
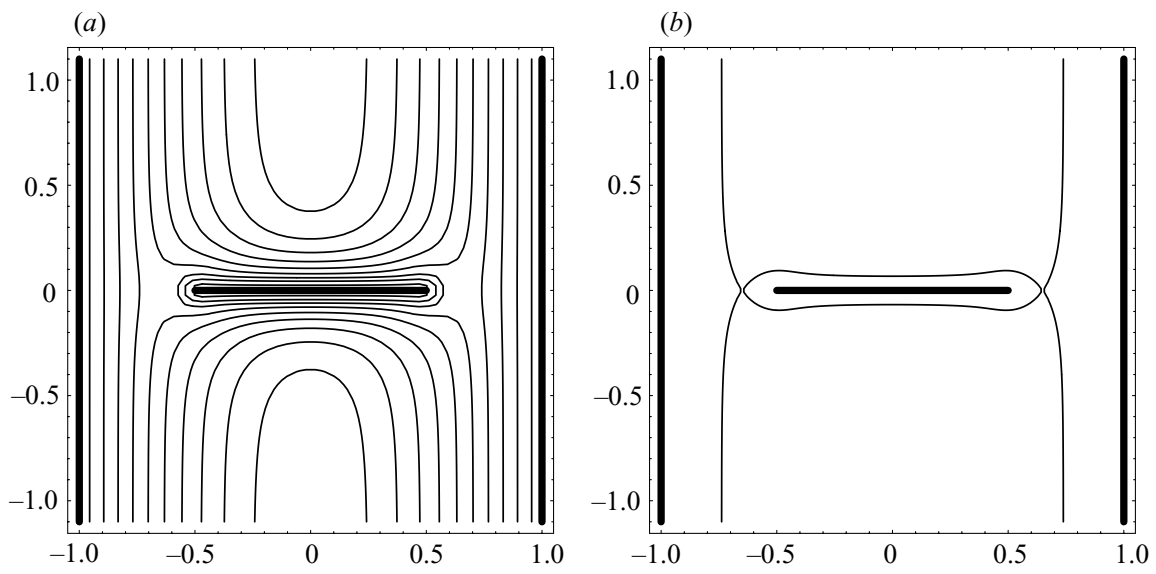

FigURE 15. An island of length 1 lying transversely centrally within a channel of width 2 . (a) Vortex trajectories. (b) The separatrix.

this is the transformation

$$
\hat{z}=G^{-1}(z)=(2 / \pi) \sin ^{-1} z, \quad z=G(\hat{z})=\sin (\pi \hat{z} / 2),
$$

which opens the angle between the headland sides from 0 to $\pi$ and so gives in the $\hat{z}$-plane an island partially blocking a channel. Figure 15 gives vortex trajectories and the separatrix for an island of length 1 lying centrally across a channel of width 2 . Vortices propagating towards the island pass by provided they are sufficiently close to a channel wall (as for vortices near offshore islands in Johnson \& McDonald $2004 a$, 2005) but are reflected if they lie on the other side of the separatrix. Vortices sufficiently close to the island orbit the island as expected.

Similarly, results have been presented so far for thin islands but extend immediately to islands of finite thickness. The transformation

$$
z=G^{-1}(\hat{z})=\frac{1}{2} k[(\hat{z} / \delta)+(\delta / \hat{z})], \quad \hat{z}=G(z)=(z \delta / k)\left[1+\sqrt{1-(k / z)^{2}}\right],
$$

leaves the headlands unchanged but maps the thin island in the $z$-plane to a circular island of radius $\delta=\left(1+\sqrt{1-k^{2}}\right) / k$ (so $\left.k=2 /(\delta+1 / \delta)\right)$ in the $\hat{z}$-plane. Figure 16 gives vortex trajectories and the separatrix for a circular island of radius 0.3 in a gap of width 2 and shows how closely the thin-island trajectories resemble those of thicker islands. The three transformations here can, of course, be combined to give asymmetrically placed, finite-width, elliptical islands lying within channels and 
(a)

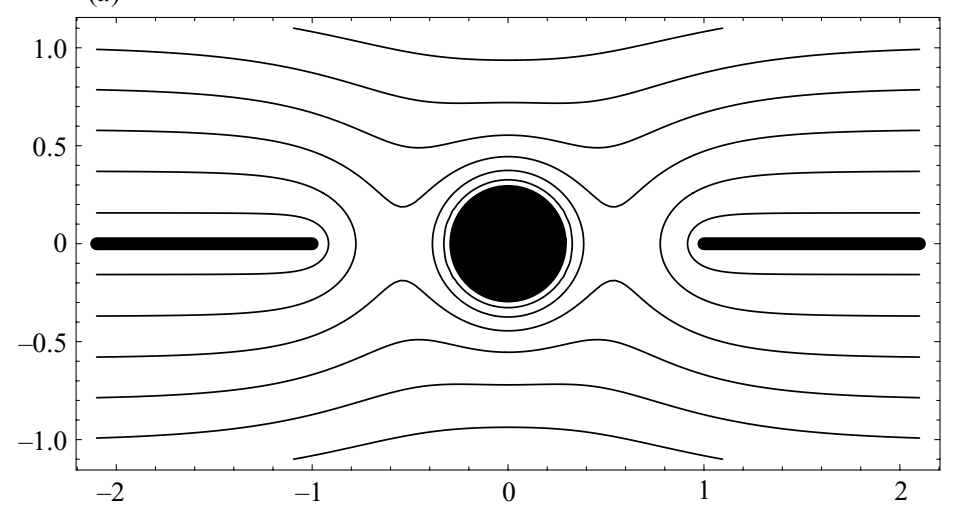

(b)

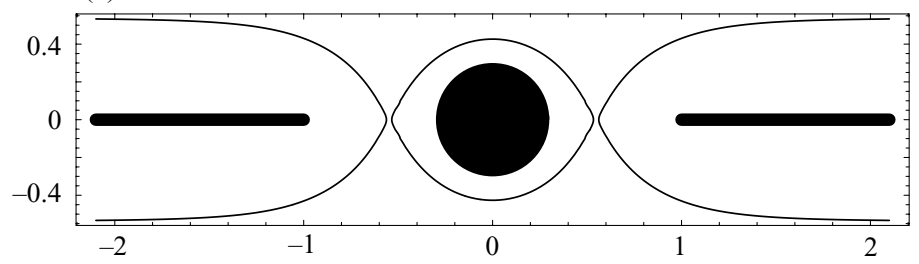

FIGURE 16. A circular island of radius 0.3 within a gap of width 2. (a) Vortex trajectories (b) The separatrix.

gaps. More complex geometries with irregularly shaped islands require the conformal mapping to simpler domain shapes to be obtained numerically, a computation that is well-established (Wegmann 2001). Once known, this mapping allows the point vortex trajectories and irrotational part of the vortex patch velocities to be found immediately.

The attached flows described here model flows past circular and narrow, but roundended, islands; however no-slip boundary conditions associated with weak horizontal friction could lead to vortex sheet shedding at the ends of sufficiently sharp-edged islands in weakly viscous flows when a vortex passes by. If the shed sheet were to extend sufficiently far from the island then the material line surrounding the island would not close at large times and the constraint of zero circulation could be broken. A circulation condition that the shed sheets departs tangentially from the island end, as for a sheet shed from an aerofoil, might then be more appropriate. Consideration of these unsteady separation effects and those of Ekman pumping would appear to require a more extensive numerical treatment.

Two important features of the three-dimensional interaction of oceanic eddies with topography have not been treated here. Following Dewar (2002) the topographic features here have been taken to have vertical sides. Dewar (2002) argues convincingly that, on the horizontal and vertical scales of a Meddy, typical oceanic barriers do indeed appear vertical. Nevertheless, sufficiently near features the finite bottom slope may have a strong effect on vortex trajectories. Although some analysis of vortices above finite-depth changes can be made when background rotation is negligible (Johnson \& McDonald 2004c) these effects remain to be fully investigated. The flow here has also been taken to be barotropic (depth-independent) yet many oceanic eddies have significant baroclinic vertical structure. Dewar (2002) uses a three-layer model so his streamfunctions for line vortices are a combination of both logarithmic 
and Bessel function terms from the Laplace (barotropic) and modified Helmholtz (baroclinic) operators. The present analysis gives the barotropic contribution to the motion immediately, but obtaining the baroclinic contribution requires a different technique and the lack of conformal invariance reduces the size of the class of topographic obstacles that can be considered. The approximate methods of Johnson \& McDonald (2005) also extend directly to multilayer flow.

Although presented above in the context of oceanic vortices, the propagation of intense vortices near partial barriers may also offer an idealized and simple model of hurricane dynamics near coasts.

Note added in proof: The closed-form results here for point vortex trajectories in doubly connected domains can be extended to higher connectivity, in the absence of background flow and for zero round-island circulations, using infinite products of mappings (Crowdy \& Marshall 2005a, $b$ ).

We are indebted to Professor Dritschel for providing us with a copy of his 1988 code which formed the basis for the code developed here. E. R. J. is also indebted to the School of Mathematical Sciences at Monash University for their hospitality while some of this work was carried out and to the UK Natural Environment Research Council through Grant NER/A/S/2000/01323 to present this work at the European Geophysical Union Assembly in April 2004.

\section{Appendix. Patch centroid motion}

The $x$-coordinate of the vorticity centroid of a patch can be defined as $x_{C}=$ $\int x Z / \int Z$, where $Z(x, y)$ is the patch vorticity and the integrals are evaluated over the support of $Z$ (i.e. the patch). The Euler equations show that $\int Z$ is a constant and give

$$
\left(\int Z\right) \dot{x}_{C}=\int x \dot{Z}=-\int x \nabla \cdot(\boldsymbol{u} Z)=-\int[\nabla \cdot(x \boldsymbol{u} Z)-Z \boldsymbol{u} \cdot \nabla x],
$$

where the overdot denotes the time derivative. The divergence in the final integrand gives a boundary integral, which vanishes for a bounded patch and impermeable boundaries, and the remaining term is simply the $x$-component, $u$, of the velocity vector $\boldsymbol{u}$. Now write $u=u_{V}+u_{e}$, where $u_{V}$ is the velocity obtained from the infinitedomain Green's function, giving the rotational velocity field induced by the patch in isolation, and $u_{e}$ is the remaining velocity field from all other patches, from images in boundaries and from any superposed irrotational flow fields. Then the usual manipulations for conservation of impulse show that $u_{V}$ makes no contribution to the final integral of (A 1). For a patch of constant vorticity $\omega, x_{C}$ becomes the geometric patch centroid and (A 1) gives

$$
\omega \mathscr{A} \dot{x}_{C}=\int u_{e} Z=\omega \int u_{e} \approx \omega \mathscr{A} u_{e}\left(x_{C}, y_{C}\right),
$$

where $\mathscr{A}$ is the (constant) patch area and the final approximation is obtained by replacing the integral by $\mathscr{A}$ times the integrand evaluated at the centroid. Since $u_{e}$ is harmonic throughout the patch this replacement is exact when the patch is circular. The analogous result holds for $y_{C}$ and so the centroid of a circular patch moves as a particle in the external velocity field $u_{e}$, as does a line vortex. Moreover for a circular patch the velocity field derived from the infinite-domain Green's function is precisely that for a point vortex with the same circulation and so induces the same images in any solid boundaries: $u_{e}$ is unaltered by replacing the patch by an equivalent line 
vortex. The patch centroid travels along the same path at the same speed as the equivalent line vortex.

\section{REFERENCES}

Abramowitz, M. \& Stegun, I. A. 1972 Handbook of Mathematical Functions. US Dept. of Commerce.

Benzi, R. \& Legras, B. 1987 Wave-vortex dynamics. J. Phys. A 20, 5125-5144.

Campbell, L. J., Doria, M. M. \& Kadtke, J. B. 1989 Energy of infinite vortex lattices. Phys. Rev. A 39, 5436-5439.

Crowdy, D. G. \& Marshall, J. S. 2005 a Analytical formulae for the Kirchhoff-Routh path function in multiply-connected domains. Proc. R. Soc. Lond. A (to appear).

Crowdy, D. G. \& Marshall, J. S. $2005 b$ The motion of a point vortex around multiple circular islands. Phys. Fluids (to appear).

DeEm, G. S. \& Zabusky, N. J. 1978 Vortex waves: stationary 'V-states', interactions, recurrence, and breaking. Phys. Rev. Lett. 40, 859-862.

Dewar, W. K. 2002 Baroclinic eddy interaction with isolated topography. J. Phys. Oceanogr. 32, 2789-2805.

Dritschel, D. G. 1988 Contour surgery: a topological reconnection scheme for extended contour integrations using contour dynamics. J. Comput. Phys. 77, 240-266.

Fratantoni, D., Johns, W. \& Townsend, T. 1995 Rings of the North Brazil Current: their structure and behaviour inferred from observations and numerical simulation. J. Geophys. Res. 100, $10633-10654$.

Helfrich, K. R., Pedlosky, J. \& Carter, E. 1999 The shadowed island. J. Phys. Oceanogr. 29, 2559-2577.

Johnson, E. R. 1989 Scattering of shelf waves by islands. J. Phys. Oceanogr. 19, 1311-1316.

Johnson, E. R. \& Clarke, S. R. 1998 Inviscid vortical boundary layers. In Numerical Methods for Fluid Dynamics (ed. M. J. Baines), vol. 6, pp. 83-97. Oxford: Will Print.

Johnson, E. R. \& MCDonald, N. R. 2004a The motion of a vortex near a gap in a wall. Phys. Fluids 16, 462-469.

Johnson, E. R. \& McDonald, N. R. $2004 b$ The motion of a vortex near two circular cylinders. Proc. R. Soc. Lond. A 460, 939-954.

Johnson, E. R. \& McDonald, N. R. 2004c Surf zone vortices over stepped topography. J. Fluid Mech. 511, 265-283.

Johnson, E. R. \& MCDonald, N. R. 2005 The point island approximation in vortex dynamics. Geophys. Astrophys. Fluid Dyn. 99, 49-60.

Lin, C. C. 1941 On the motion of vortices in two-dimensions. I. Existence of the Kirchhoff-Routh function. Proc. Natl Acad. Sci. 27, 570-577.

McWilliams, J. C. 1985 Submesoscale, coherent vortices in the ocean. Rev. Geophys. 23, 165-182.

NoF, D. 1995 Choked flows from the Pacific to the Indian Ocean. J. Phys. Oceanogr. 25, 1369-1383.

O’NeIL, K. A. 1989 On the Hamiltonian dynamics of vortex lattices. J. Math. Phys. 30, 1373-1379.

Pedlosky, J. 1994 Stratified abyssal flow in the presence of fractured ridges. J. Phys. Oceanogr. 24, 403-417.

Pedlosky, J. 2000 The transmission of Rossby waves through basin barriers. J. Phys. Oceanogr. 30, 495-511.

Pedlosky, J. 2001a Steady baroclinic flow through ridges with narrow gaps. J. Phys. Oceanogr. 31, $2418-2440$.

Pedlosky, J. $2001 b$ The transparency of ocean barriers to Rossby waves: The Rossby slit problem. J. Phys. Oceanogr. 31, 336-352.

Pedlosky, J., Pratt, L. J., Spall, M. A. \& Helfrich, K. R. 1997 Circulation around islands and ridges. J. Mar. Res. 55, 1199-1251.

Pedlosky, J. \& Spall, M. 1999 Rossby normal modes in basins with barriers. J. Phys. Oceanogr. 29, 2332-2349.

Pratt, L. J. \& Spall, M. A. 2003 A porous-medium theory for barotropic flow through ridges and archipelagos. J. Phys. Oceanogr. 33, 2702-2718. 
Richardson, P. L. \& Tychensky, A. 1998 Meddy trajectories in the Canary Basin measured during the SEMAPHORE experiment, 1993-1995. J. Geophys. Res. 103 (C11), 25029-25045.

Routh, E. J. 1881 Some applications of conjugate functions. Proc. Lond. Math. Soc. 12, 73-89.

Saffman, P. G. 1992 Vortex Dynamics. Cambridge University Press.

Sheremet, V. A. 2001 Hysteresis of a western boundary current leaping across a gap. J. Phys. Oceanogr. 31, 1247-1259.

Simmons, H. L. \& Nof, D. 2002 The squeezing of eddies through gaps. J. Phys. Oceanogr. 32, 314-335.

Stremler, M. A. \& Aref, H. 1999 Motion of three point vortices in a periodic parallelogram. J. Fluid Mech. 392, 101-128.

Tkachenko, V. K. 1966 On vortex lattices. Sov. Phys. JETP 22, 1282-1286.

WANG, G. H. \& DewaR, W. K. 2003 Meddy-seamount interactions: Implications for the Mediterranean salt tongue. J. Phys. Oceanogr. 33, 2446-2461.

Wegmann, R. 2001 Fast conformal mapping of multiply connected regions. J. Comput. Appl. Math. 130, 119-138.

Weiss, J. B. \& McWilliams, J. C. 1991 Nonergodicity of point vortices. Phys. Fluids A 3, 835-844.

Wells, J. R. \& Helfrich, K. R. 2001 Circulation around a thin zonal island. J. Fluid Mech. 437, 301-323.

Whittaker, E. T. \& Watson, G. N. 1927 A Course of Modern Analysis. Cambridge University Press.

Zabusky, M. H., Hughes, K. V. \& Roberts, K. V. 1979 Contour dynamics for the Euler equations in two-dimensions. J. Comput. Phys. 30, 96-106. 